\title{
RECOMENDAÇÕES TÉCNICAS PARA 0 REGISTRO DO ELETRENCEFALOGRAMA (EEG) NA SUSPEITA DA MORTE ENCEFÁLICA
}

\author{
FRANCISCO JOSÉ C. LUCCAS*, NADIA I. O. BRAGA*, CARLOS EDUARDO SOARES SILVADO*
}

\begin{abstract}
RESUMO - Neste trabalho, desenvolvido por uma comissão nomeada pela Sociedade Brasileira de Neurofisiologia Clínica, são apresentadas as recomendações referentes ao registro do eletrencefalograma (EEG) nos casos de suspeita de morte encefálica, enfatizando que, apesar do necessário respeito aos parâmetros técnicos, o método não visa substituir o exame neurológico, mas complementá-lo.
\end{abstract}

PALAVRAS-CHAVE: EEG, morte encefálica, recomendações.

\section{Guidelines for electroencephalogram (EEG) recording in suspected brain death}

ABSTRACT - Brazilian Clinical Neurophysiology Society guidelines and pertaining comments concerning electroencephalogram (EEG) recording in suspected brain death are presented. EEG is not intended as a substitute, rather as a complement to neurologic evaluation.

KEY WORDS: EEG, brain death, guidelines.

Os requisitos mínimos para o registro do EEG na suspeita da morte encefálica foram inicialmente estabelecidos por comitê "ad hoc" da "American EEG Society", refletindo o estágio do desenvolvimento no final dos anos 60. Nessa ocasião, estudos feitos revelaram que, de 2650 casos de coma profundo com EEG presumivelmente "isoelétrico", apenas três mostraram recuperação da função encefálica e todos estes três pacientes haviam recebido doses muito elevadas de medicação depressora do $\mathrm{SNC}^{1-3}$. Muitos dos registros inicialmente citados como isoelétricos foram, após revisão cuidadosa, considerados apenas como registros de baixa voltagem ou obtidos através de parâmetros técnicos inadequados ${ }^{4-6}$. O comitê propôs a eliminação de termos não fisiológicos como "isoelétrico" ou "linear" e, da mesma forma, recomendou que palavras como "plano" ou "chato" não fossem usadas. Foi sugerido o nome silêncio elétrico cerebral (SEC) $)^{1-3}$. Subsequentemente, inatividade elétrica cerebral foi o termo recomendado no glossário da Federação Internacional das Sociedades de EEG e Neurofisiologia Clínica. Os dois termos têm sido considerados sinônimos?

Atualmente, o registro do EEG para auxiliar na determinação da morte encefálica não está mais limitado aos grandes laboratórios, pois muitos hospitais menores possuem unidades de tratamento intensivo e equipamentos de EEG; além disso, a instrumentação do EEG está substancialmente melhorada e muitos serviços possuem vários anos de experiência nessa área. Dessa forma, impõe-se a necessidade de divulgar as normas básicas para este procedimento, visando inclusive uniformizar critérios.

É importante considerar que o registro do EEG para o diagnóstico de morte encefálica deve ser cercado de grandes cuidados, porque sempre é consistentemente mais difícil afirmar uma ausência

*Comissão nomeada pela Sociedade Brasileira de Neurofisiologia Clínica (SBNC) para elaboração das recomendações referentes ao registro do EEG na suspeita de morte encefálica. Aceite: 11-maio-1998. 
do que uma presença. Para se afirmar a presença da atividade elétrica é suficiente encontrá-la. Entretanto, se não for encontrada a atividade elétrica, deve-se ter maiores cuidados para garantir a sua ausência; um falso resultado de ausência poderia, por exemplo, decorrer de procura: em local errado, de modo errado ou por um tempo inadequado ${ }^{8}$.

Finalmente, deve-se ressaltar que ainda hoje a transmissão por telefone do EEG não é recomendada para a determinação do silêncio elétrico cerebral, por causa do ruído elétrico imprevisível e inerente existente nas redes telefônicas, especialmente quando comparado ao sinal muito baixo passível de ser encontrado no silêncio elétrico cerebral ou próximo a ele? .

\section{Definição de Silêncio Elétrico Cerebral (SEC)}

A inatividade elétrica cerebral ou silêncio elétrico cerebral (SEC) pode ser definido como a ausência de atividade elétrica no EEG acima de $2 \mu \mathrm{V}$ (microvolts), quando registrada por pares de eletrodos colocados no couro cabeludo a distâncias de 10 ou mais centímetros e com impedâncias intereletrodos abaixo de 5000 a 10000 ohms e acima de 100 ohms.

Nessa definição enfatiza-se a voltagem mínima (notar que ela é dependente do equipamento usado), o uso de maiores distâncias entre os eletrodos e reafirma-se a importância de colocar os eletrodos com impedâncias adequadas ${ }^{9,10}$.

\section{Recomendações para o registro do EEG na suspeita de morte encefálica}

São apresentadas 10 recomendações para o registro de EEG em casos de suspeita de morte encefálica, com as respectivas justificativas e comentários pertinentes ${ }^{8-11}$.

1. Colocar sobre o couro cabeludo todos os eletrodos (20), de acordo com o sistema internacional 10-20 de colocação de eletrodos em eletrencefalografia.

Todas as áreas cerebrais devem ser cobertas, para que se tenha certeza de que a ausência de atividade não representa apenas um fenômeno focal. O uso de instrumentos com apenas um ou dois canais, como aqueles utilizados na monitoração pelo EEG dos níveis de anestesia, é inaceitável para se determinar o SEC.

Há uma recomendação antiga citando um mínimo de oito eletrodos, cobrindo apenas as áreas frontais, centrais, temporais e occipitais; porém, considerando a importância da decisão de morte encefálica, não se julga adequada a supressão de certos eletrodos, com cobertura mínima de algumas áreas. Além disso, como não se pode saber "a priori” que será obtido um registro de SEC e, visando estar preparados para qualquer situação, será sempre aconselhável usar o conjunto completo de eletrodos no couro cabeludo, do mesmo modo que no exame de rotina; esse conjunto completo de eletrodos deve incluir as posições de linha média (Fz, $\mathrm{Cz}$ e Pz), além dos fronto-polares, frontais, centrais, parietais, occipitais e temporais. Um eletrodo de terra (referência) deve ser usado, colocado no corpo do paciente; contudo, para registros feitos em UTI, se já houver um fio de terra de qualquer outro equipamento elétrico instalado no paciente, esse eletrodo pode não ser colocado; nota: não confundi-lo com o fio terra das instalações elétricas ${ }^{9,10}$.

2. As impedâncias dos eletrodos devem estar abaixo de 5000 a 10000 ohms, porém acima de 100 ohms.

a) As situações caracterizadas por atividade elétrica cerebral de baixa voltagem e instrumento de alta sensibilidade requerem aplicação bastante escrupulosa dos eletrodos. Desta forma, impedâncias desbalanceadas (valores muito diferentes no par de eletrodos) podem distorcer o EEG. Isto pode ocorrer mesmo se as impedâncias dos dois eletrodos estiverem dentro da faixa recomendada; se um eletrodo mostrar um valor de impedância relativamente alto (por exemplo, $8000 \mathrm{ohms}$ ), quando comparado com a do segundo eletrodo do par (por exemplo, $2000 \mathrm{ohms),} \mathrm{o} \mathrm{amplificador} \mathrm{torna-se}$ desbalanceado e tende a ampliar irregularmente os sinais externos; será facilitado o registro da interferência de $60 \mathrm{~Hz}$ ou de outros artefatos. 
b) Há uma queda acentuada dos potenciais se as impedâncias estiverem abaixo de $100 \mathrm{ohms}$ e, naturalmente, nenhum potencial com zero ohms. É essencial que um eventual excesso de pasta condutora não se espalhe entre dois eletrodos, criando uma ponte salina, que atenuará o sinal registrado entre esse par de eletrodos. Esta ocorrência poderia determinar um registro falso de SEC. Portanto, deve-se fazer uma acurada medição das impedâncias durante o registro para se assegurar de que elas são da magnitude adequada.

c) Apesar de não serem recomendados para uso de rotina, eletrodos subdérmicos de agulha têm sido usados com sucesso em registros de suspeita de morte encefálica. Têm a vantagem de serem instalados com maior rapidez. Estes eletrodos subdérmicos devem ser colocados todos com a mesma orientação. Embora mostrem impedâncias mais altas, os seus valores tendem a ser similares ${ }^{9,10}$.

\section{Deve-se testar a integridade de todo o sistema de registro.}

A calibração instrumental rotineira testa a operação dos amplificadores e dos inscritores, mas não exclui a possibilidade de uma ponte salina ou de um circuito aberto nos eletrodos, na caixa de eletrodos, no cabo ou na entrada do aparelho. Se o registro do EEG sugerir SEC, deve-se testar a integridade do sistema tocando-se suavemente cada eletrodo da montagem com a ponta de um lápis ou de um cotonete, para criar um artefato. Esse teste permite verificar que a caixa de eletrodos está conectada ao aparelho; registros feitos com a caixa de eletrodos inadvertidamente desconectada poderiam às vezes assemelhar-se ao de um EEG de baixa amplitude. O teste também demonstra que o posicionamento do seletor combina com o posicionamento programado dos eletrodos ${ }^{9,10}$.

\section{A distância intereletrodos deve ser no mínimo de $10 \mathrm{~cm}$ (usar montagem modificada com} maiores distâncias ou com distâncias duplas entre os eletrodos).

No sistema internacional 10-20 a distância média intereletrodos em adultos é, em geral, de 6$6,5 \mathrm{~cm}$. Sabe-se que a diferença de potencial registrada entre o par de eletrodos aumenta com sua separação, atingindo um valor máximo por volta de $10 \mathrm{~cm}$. Um registro obtido com as distâncias habituais intereletrodos e com a sensibilidade de rotina poderia até sugerir SEC. Contudo, para maior segurança, o registro deverá ser feito usando as maiores distâncias entre eletrodos, pois haverá maior possibilidade de captar e visualizar no traçado os potenciais cerebrais. Dessa forma, ao se utilizarem montagens transversais ou longitudinais bipolares, são recomendadas derivações com distância dupla dos eletrodos, por exemplo: F3 - P3, C3 - O1, Fp1 - T3, F7 - T5, etc.

Lembrar que o registro com referência na orelha é quase invariavelmente muito contaminado pelos potenciais cardíacos (EKG); neste caso, a referência em $\mathrm{Cz}$ poderia ser mais satisfatória. As regiões posteriores e occipitais podem ser particularmente susceptíveis aos artefatos de movimento induzidos pela respiração artificial, o que também complicaria seu uso como referência comum.

Obedecendo ao princípio das maiores distâncias (distâncias duplas) intereletrodos, podem ser recomendadas as seguintes montagens para se usar na suspeita da morte encefálica:

1. para 16 canais: F3-P3; C3-O1; Fp1-T3; F7-T5; F4-P4; C4-O2; Fp2-T4; F8-T6; F7-Fz; Fz-F8; T3-Cz; Cz-T4; T5-Pz; Pz-T6; 2 eletrodos no dorso da mão direita, para funcionar como monitor ambiental; 2 eletrodos no precórdio, para avaliar os potenciais cardíacos (EKG). As derivações 1 a 8 compreendem montagem longitudinal bipolar com distâncias duplas intereletrodos; de 9 a 14 são utilizadas montagens transversais (anterior, média e posterior) também com distâncias duplas; no canal 15 coloca-se o monitor ambiental e no 16 o registro dos potenciais cardíacos (EKG).

2. para 8 canais: F3-P3; C3-O1; Fp1-T3; F7-T5; F4-P4; C4-O2; Fp2-T4; F8-T6. Eventualmente podem ser sacrificados os canais com eletrodos fronto-polares, substituindo-os pelo monitor ambiental e pelo EKG. Naturalmente, o registro em 16 canais permite avaliação mais completa.

Os 2 eletrodos no dorso da mão direita (onde não há atividade elétrica cerebral) são colocados a 6-7 cm de distância e funcionam como monitor ambiental, permitindo conhecer as diferentes interferências sobre o registro e avaliar sua origem extracerebral ${ }^{8-10}$. 
5. A sensibilidade deve ser sempre aumentada (de um valor rotineiro de $7 \mu \mathrm{V} / \mathrm{mm}$ ) para pelo menos $2 \mu \mathrm{V} / \mathrm{mm}$ durante a maior parte do registro; fazer o registro da calibração mostrando esse valor máximo da sensibilidade.

a) Este é sem dúvida um dos parâmetros mais importantes para avaliar o SEC. Deve-se compreender que, com a sensibilidade usada rotineiramente de $7 \mu \mathrm{V} / \mathrm{mm}$ não se pode visualizar um sinal de apenas $2 \mu \mathrm{V}$, porque a linha de tinta no papel tem em média $1 / 4 \mathrm{~mm}$ de largura, isto é, do mesmo tamanho do sinal que se deseja ver. Obviamente, uma voltagem limite de $2 \mu \mathrm{V}$ (ver definição de SEC) moverá a pena por somente $1 \mathrm{~mm}$, se a sensibilidade for de $2 \mu \mathrm{V} / \mathrm{mm}$. Este sinal de $2 \mu \mathrm{V}$ será portanto melhor visualizado com uma sensibilidade de 1,5 ou $1 \mu \mathrm{V} / \mathrm{mm}$. Contudo, mesmo com essa sensibilidade pode ainda ser difícil de se ver uma atividade muito lenta que apresente uma fase ascendente da onda muito gradual. Os equipamentos atuais permitem registros prolongados com sensibilidade de 1,5 ou $1 \mu \mathrm{V} / \mathrm{mm}$. Esse aumento de sensibilidade, da ordem de 50 a $100 \%$, permite um julgamento mais confiável da presença ou da ausência de um sinal de $2 \mu \mathrm{V}$.

b) São essenciais os procedimentos apropriados e adequados de calibração. Representa boa prática calibrar com um sinal próximo do valor do sinal do EEG que se pretende registrar; assim sendo, no caso do SEC, pode ser apropriada uma calibração de 2 ou $5 \mu \mathrm{V}$. Um sinal de calibração de $50 \mu \mathrm{V}$ com sensibilidade de 1 ou $2 \mu \mathrm{V} / \mathrm{mm}$ é inútil, pois ocorre bloqueio das penas.

c) Há referências de que períodos de SEC de até 20 minutos podem raramente ocorrer em registros de baixa voltagem; consequentemente, alguns autores sugerem que o registro deve durar pelo menos 30 minutos, para ter certeza de que não se perdeu uma atividade cerebral intermitente de baixa voltagem. Entretanto, esta é uma situação bastante incomum e mais voltada ao registro do EEG de prematuros ou de recém-nascidos patológicos. Em adultos, se for afastado o uso de drogas depressoras do sistema nervoso central, esse critério pode ser bastante discutido. Os registros iniciais para diagnóstico do SEC chegavam a durar 2 horas; ulteriormente, o tempo foi abreviado para 1 hora e depois para 30 minutos. Essa redução foi baseada no critério de que, "se nos primeiros 30 minutos ocorresse o SEC, nunca havia sido observada qualquer atividade nos 30 minutos seguintes". Esse raciocínio poderia ser estendido até para os 5 minutos iniciais ou mesmo para 1 minuto ! No entanto, o registro do EEG se baseia em amostragem e são bem conhecidos os riscos de amostras muito reduzidas. $\mathrm{Na}$ verdade, não foram realizados estudos prospectivos visando determinar qual o tempo adequado de registro e, por enquanto, seria sensato adotar uma atitude mais conservadora, sempre apoiada nos dados clínicos. Recomenda-se obter um registro contínuo por pelo menos 30 minutos $^{8-11}$.

\section{Os filtros devem ser usados de forma judiciosa na avaliação do SEC.}

Para se evitar atenuação tanto de atividade rápida como de atividade lenta de baixa voltagem, sempre que possível, não se deve colocar os filtros de alta frequência abaixo de $30 \mathrm{~Hz}$ e os filtros de baixa frequência acima de $1 \mathrm{~Hz}$. Sabe-se que constantes de tempo curtas (ou filtros de baixa frequiência elevados) atenuam os potenciais lentos e, na situação próxima ao SEC, pode haver um predomínio de potenciais nas faixas lentas, delta principalmente; por essa razão, deve-se evitar a atenuação dessas frequências. Recomenda-se manter os filtros entre 0,5-70 Hz pelo menos em parte do registro; o filtro de baixa frequiência de $0,5 \mathrm{~Hz}$ equivale a uma constante de tempo de $0,3 \mathrm{~s}$.

Pode-se usar sem receio o filtro de entalhe (ou de corte) de $60 \mathrm{~Hz}$, que corta as frequências na faixa de $60 \mathrm{~Hz}$; entretanto, com boas instalações elétricas (bom fio terra na UTI), frequentemente não há necessidade de aplicar esse filtro especial ${ }^{9-10}$.

7. Técnicas adicionais de monitoração devem ser empregadas quando necessárias.

No registro do EEG ocorre a captação de um somatório de ondas cerebrais verdadeiras, de outros sinais fisiológicos e de artefatos (tanto internos como externos ao aparelho e de origem mecânica, eletromagnética e/ou eletrostática). Naturalmente, quando a sensibilidade é aumentada os artefatos são também acentuados e, consequentemente, devem ser identificados, para que se possa avaliar adequadamente se o EEG (atividade elétrica cerebral) está ou não presente. Deve-se enfatizar que o mais seguro contra muitos tipos de artefatos é um sistema estável de eletrodos com baixa impedância. 
a) Como muito raramente se vê um registro de SEC sem grandes quantidades de artefatos de EKG (potenciais cardíacos), um monitor de sinais cardíacos será importante.

b) Se o artefato produzido pelo respirador não puder ser eliminado, ele deverá ser documentado através de anotação no registro (pela técnica) ou então ser monitorizado por meio de transdutores. Pode-se identificar com segurança este artefato desconectando por curto período (menor de 10 segundos) o respirador.

c) Frequentemente necessita-se de um monitor adicional para outros artefatos originados do paciente ou induzidos pelo meio ambiente; recomenda-se colocar um par de eletrodos no dorso da mão direita, separados por 6 a $7 \mathrm{~cm}$.

d) Sabe-se que contaminações por potenciais musculares podem persistir em pacientes com registro de SEC. Se os potenciais musculares forem de tal amplitude que impeçam a visualização do traçado, pode ser necessário reduzi-los ou eliminá-los com o uso de um agente bloqueador neuromuscular como Pavulon (brometo de pancurônio) ou Quelicin (succinilcolina). Este procedimento deve ser efetuado sob a orientação de um anestesista ou de outro médico familiarizado com o uso da droga.

e) O ruído do aparelho e a interferência externa podem ser convenientemente checados por um "paciente falso", isto é, um resistor de 10000 ohms entre a entrada G1 e a entrada G2 de um canal.

f) Contudo, mesmo com técnica adequada, registrar um EEG com a sensibilidade elevada anteriormente especificada pode às vezes deixar o eletrencefalografista em consideráveis dificuldades. Nesse caso, deve-se tentar determinar qual parte do registro é resultante de sinais fisiológicos nãocerebrais ou é resultante de artefatos não-fisiológicos, inclusive no nível constante de ruído do sistema completo na UTI (indicado, por exemplo, pelo registro do dorso da mão); a seguir, deve-se fazer uma estimativa se a atividade restante (EEG) excede ou não $2 \mu \mathrm{V}$ em amplitude. Se isso não puder ser feito de modo confiável, o relatório do EEG deve indicar claramente as incertezas e o registro não poderá ser definitivamente classificado como demonstrando SEC ${ }^{9-11}$.

8. Não deve haver reatividade no EEG a estímulos sômato-sensitivos, auditivos ou visuais, mesmo intensos.

No estudo colaborativo norte-americano não ocorreram situações com reatividade a estímulos em registros de pacientes com SEC. Qualquer atividade no EEG que pareça resultante dos estímulos acima citados ou de quaisquer outros (lembrar que aspiração das vias respiratórias ou outros procedimentos de enfermagem podem representar estímulos potentes) tem que ser cuidadosamente distinguida dos sinais fisiológicos não-cerebrais e dos artefatos não-fisiológicos.

A estimulação pode também auxiliar no estabelecimento do grau de reatividade dos registros que não forem caracterizados como SEC.

Podem ser citados como exemplos de estímulos usados de rotina: auditivo (chamar o nome, palmas, etc.); visual (abertura passiva dos olhos, estimulação luminosa intermitente, etc.); sômatosensitivo (doloroso, táctil, etc.). Durante certa época também era recomendada de rotina a compressão dos globos oculares, visando observar se ocorria diminuição de freqüência cardíaca através de reflexos vagais, a mostrar integridade de vias reflexas no tronco cerebral. Recomenda-se que o serviço padronize e utilize sempre os mesmos estímulos na avaliação do SEC; por exemplo: palmas, abertura / fechamento passivo dos olhos, doloroso.

Uma outra maneira mais especializada para avaliar as respostas do sistema nervoso a estímulos consiste em executar os potenciais evocados visuais, auditivos e sômato-sensitivos; notar que, qualitativa e quantitativamente, o registro dos potenciais evocados não pode ser considerado igual à avaliação da reatividade no EEG e, portanto, os métodos não deveriam ser excludentes ${ }^{9-10}$.

\section{Os registros devem ser feitos por pessoal técnico qualificado.}

A adequada competência é essencial ao se registrar casos com suspeita de SEC. Os registros são frequentemente feitos em circunstâncias difíceis e incluem muitas possíveis fontes de artefatos. 
A eliminação da maioria dos artefatos e a identificação de outros deve ser executada por um técnico qualificado. É necessário treinamento específico dirigido a esta área, que vai nitidamente além do treinamento geral obrigatório; o registro do EEG na suspeita do SEC não é um registro que possa ser obtido por pessoal técnico não qualificado.

O técnico deve trabalhar sob a direção de um neurofisiologista clínico com área de atuação em eletrencefalografia. E o médico deve estar presente na ocasião do registro. Também é muito importante a qualificação do especialista, pois nem todos os médicos com atuação na área de eletrencefalografia têm a adequada experiência do trabalho em situações de terapia intensiva ou de sala de urgência ${ }^{10}$.

\section{A repetição do EEG somente deve ser efetuada se houver dúvidas clínicas ou dúvidas} sobre a existência de SEC.

No estudo colaborativo norte-americano sobre a morte encefálica não houve paciente que sobrevivesse depois que o EEG apresentou SEC, desde que hipotermia ou superdosagem de drogas depressoras do sistema nervoso central fossem excluídas. Como regra geral, uma vez detectada a morte encefálica utilizando os critérios clínicos neurológicos e com EEG confirmatório, ocorre falência cárdio-circulatória em curto prazo, que vai de horas a poucos dias; sobrevindo essa falência, logo ocorre parada cardíaca. Entretanto, como toda regra, essa também comporta exceções e há descrição de sobrevivência somática prolongada (até cerca de 2 e meio meses) após a ocorrência de morte encefálica ${ }^{12}$.

Existe atualmente bastante experiência para se afirmar que, em adultos e em crianças acima de 2 anos de idade, um único EEG apresentando SEC, na vigência do quadro neurológico compatível, pode representar procedimento altamente confiável na determinação da morte encefálica ${ }^{13,14}$. Portanto, a repetição do EEG deve somente obedecer a critérios clínicos e o intervalo não deve ser preestabelecido, a não ser levando em consideração todos os dados disponíveis. Não há necessidade de se repetir rotineiramente o EEG para estabelecer o diagnóstico de morte encefálica. Também é importante enfatizar que o EEG, como exame complementar, seguramente não pode substituir o exame clínico neurológico ${ }^{10-11}$.

\section{REFERÊNCIAS}

1. Report of the Ad Hoc Committee of the Harvard Medical School to Examine the Definition of Brain Death. A definition of irreversible coma. JAMA 1968;205:337-340.

2. Silverman D, Saunders MG, Schwab RS, Masland RL. Cerebral death and the electroencephalogram: report of the Ad Hoc Committee of the American Electroencephalographic Society on EEG Criteria for Determination of Cerebral Death. JAMA 1969;209:1505-1510.

3. Silverman D, Masland RL, Saunders MG, Schwab RS. Irreversible coma associated with electrocerebral silence. Neurology 1970;20:525-533.

4. Bird TD, Plum F. Recovery from barbiturate overdose coma with a prolonged isoelectric electroencephalogram. Neurology 1968;18:456-460.

5. Green JB, Lauber A. Return of EEG activity after electrocerebral silence: two case reports. J Neurol Neurosurg Psychiatry 1972;35:103-107.

6. Jorgensen EO. The EEG during severe barbiturate intoxication. Acta Neurol Scand (Suppl 43) 1970;46:281.

7. Chatrian GE, Bergamini L, Dondey M, Klass DW, Lennox-Buchtal M, Petersen I. A glossary of terms most commonly used by clinical electroencephalographers. Electroenceph Clin Neurophysiol 1974;37:538-548.

8. Henry CE. Optimal display of EEG activity. In Klass DW, Daly DD (eds). Current practice of clinical electroencephalography. New York: Raven Press 1979; 204-220.

9. American Electroencephalographic Society Committee. Guideline Three: Minimum technical standards for EEG recording in suspected cerebral death. J Clin Neurophysiol 1986;3(Suppl 1):12-17.

10. Luccas FJC, Lopes JA, Caivano ABS, Lourenço FMR, Silva MMR, Silva MLI. Monitorização eletrofisiológica. In Stávale MA (ed). Bases da terapia intensiva neurológica. São Paulo: Livraria Santos Editora, 1996;482-506.

11. Kaufman HH, Lynn J. Brain death. Contemp Neurosurg 1989;11:2-6.

12. Parisi JE, Kim RC, Collins GH, Hilfinger MF. Brain death with prolonged somatic survival. N Engl J Med 1982;306:14-16.

13. Alvarez LA, Moshé SL, Belman AL, Maytal J, Resnick TJ, Keilson M. EEG and brain death determination in children. Neurology 1988;138:227-230.

14. Report of Special Task Force. Guidelines for the determination of brain death in children. Pediatrics 1987;80:298-300. 